\title{
Impact of an Interprofessional, Longitudinal, Undergraduate Student-Initiated Home Visit Program Towards Interprofessional Education
}

\section{Kennedy $\mathrm{Ng}$}

National Cancer Centre Singapore https://orcid.org/0000-0001-6630-3803

Gloria Yao Chi Leung

Singapore General Hospital

Ka Shing Yow

National University Hospital Singapore

Nerice Heng Wen Ngiam

Singapore General Hospital

Dillon Guo Dong Yeo

Singapore General Hospital

Angeline Jie-Yin Tey

Tan Tock Seng Hospital

Melanie Si Rui Lim

KK Women's and Children's Hospital

\section{Aaron Kai Wen Tang}

Institute of Mental Health

Bi Hui Chew

Tan Tock Seng Hospital

Celine Tham

Ng Teng Fong General Hospital

Jia Qi Yeo

National Healthcare Group Polyclinics

Tang Ching Lau

National University Health System

Sweet Fun Wong

Khoo Teck Puat Hospital

Chek Hooi Wong

Khoo Teck Puat Hospital

Gerald Choon-Huat Koh ( $\nabla$ ephkohch@nus.edu.sg )

https://orcid.org/0000-0002-6453-6897

Research article

Keywords: Interprofessional, collaboration, undergraduate, longitudinal, home visit, education 
Posted Date: May 1st, 2020

DOI: https://doi.org/10.21203/rs.3.rs-23744/v1

License: (c) (i) This work is licensed under a Creative Commons Attribution 4.0 International License. Read Full License

Version of Record: A version of this preprint was published at The Asia Pacific Scholar on October 4th, 2022. See the published version at https://doi.org/10.29060/TAPS.2022-7-4/OA2785. 


\section{Abstract \\ Background}

Interprofessional (IP) practice is an essential aspect of healthcare to meet the challenges of an aging population. Tri-Generational HomeCare (TriGen) is an undergraduate student-initiated, longitudinal, IP home visit program focused on older patients with frequent hospital readmissions.

\section{Methods}

Teams comprising healthcare undergraduates and secondary school (SS) students performed home visits for frequently admitted patients (three or more visits in past six months) on a fortnightly basis over a period of six months. They were supported and trained by healthcare professionals from Khoo Teck Puat Hospital (KTPH), a regional healthcare system, and North West Community Development Council (NWCDC), a social service organisation. A multimethod approach was used, with the administration of Readiness for Interprofessional Learning Scale (RIPLS) pre- and post-intervention, and gathering post-intervention quantitative and qualitative feedback.

\section{Results}

$80.1 \%$ of 226 undergraduate participants from 2015 to 2018 enrolled in the study. There was a significant decrease in RIPLS total score from a mean of 80.3 pre-intervention to 79.0 post-intervention with a mean difference of $1.3(-2.5--0.089, p=0.035)$. Subscale analysis revealed a significant increase in the subscale "Roles and Responsibility" with a mean difference of $0.46(0.14-0.77, p<0.01)$. There was no significant change in the subscale "Teamwork" and a significant decrease in the subscale "Professional Identity" with a mean difference of $-0.14(-2.1--0.77, p<0.01)$. This was discordant to the participants' feedback. $91.6 \%$ of respondents agreed they could "better appreciate the importance of IP collaboration (IPC) in the care of patients". Qualitative analysis revealed takeaways including learning from and teaching one another, understanding one's own role and the role of other healthcare professionals, appreciating teamwork and developing friendship with undergraduates from other professions.

\section{Conclusion}

We demonstrated the feasibility of an IP, longitudinal, undergraduate-initiated home visit program that may improve attitudes towards one's role and responsibilities in IP care for future IPC.

\section{Background}

There is increasing recognition of the need for interprofessional collaboration (IPC), echoed by the World Health Organisation (WHO) in a position paper in 2010.(1) Effective collaborative practices have been shown to yield better health outcomes for patients across different populations and care settings.(2-5) Interprofessional education (IPE) aims to prepare healthcare professions for effective collaboration, and occurs when two or more professions learn about, from and with each other.(1) Research has shown that prior positive IP experiences allow 
for future positive IPC.(6) Moreover, it has been recommended for IPE to be introduced early in undergraduate healthcare courses as this may avoid stereotypes forming. $(7,8)$

Singapore faces rapidly rising healthcare costs and an aging population.(9) A small group of patients who are frequent admitters (FA), defined by Singapore's Ministry of Health (MOH) as having three or more inpatient admissions in a year, account for a disproportionate amount of the healthcare cost. The annual average cost is SGD 30,000 per FA patient compared to SGD 2,400 per non-FA patient with chronic disease.(10) Many of these patients are older, have more physical and psychiatric comorbidities, polypharmacy, and are of a lower socioeconomic strata.(11) To address their complex health and social needs, an interprofessional team approach is needed. In the United States (US), interprofessional home visit teams targeting communities with high utilisation of healthcare resources are called "hotspotters".(12,13) Equivalent programs in Singapore include the Aging-in-Place program (AIP) and Hospital-to-Home program $(\mathrm{H} 2 \mathrm{H})$.(14)

“Hotspotting" experience may allow students to understand, learn and contribute to patient's care in the community.(15) Tri-Generational HomeCare (TriGen) was conceptualised in 2014 with the following aims (some similar to the aims described by the Interprofessional Student Hotspotting Learning Collaborative(16)): 1) Reduce ageism; 2) Provide a unique opportunity for students to experience complex needs of these patients in their homes/community; 3) Increase students' awareness of the role of social determinants of health and their ability as future clinicians to impact those determinants; 4) Enhance student's ability to collaborate in interprofessional teams; 5) Develop leadership and mentoring skills.

This study aims to evaluate the effectiveness of TriGen, an IP, longitudinal, undergraduate-initiated, home-based service-learning program focused on older patients with frequent hospital readmissions in improving attitudes towards IPE amongst healthcare undergraduates.

\section{Methods}

\section{Program Design}

TriGen leverages on IP healthcare teams to tackle the challenges of an aging population. Based on the servicelearning model of dual objectives of service to the community and learning by the participants $(17,18)$, TriGen aims to provide holistic care to frequently admitted older persons and a structured learning experience for both the healthcare undergraduates and SS students.

TriGen is a collaboration between the National University of Singapore Yong Loo Lin School of Medicine (NUS YLLSoM), Khoo Teck Puat Hospital (KTPH), a regional hospital situated in the Northern part of Singapore, and North West Community Development Council (NWCDC), a grassroots organisation based in the northwest district of Singapore. KTPH developed AIP to reduce readmission rates amongst those admitted into hospital three or more times over six-months. TriGen involves participants across three generations: i) older patients enrolled in AIP, ii) healthcare undergraduate volunteers enrolled in Medicine, Nursing, Pharmacy, Social Work, Physiotherapy or Occupational Therapy course; and iii) SS student volunteers, mostly from schools in the northwest district. Each team comprises two to three undergraduates from different healthcare disciplines, and two to three SS students. 
The program begins with participants undergoing didactic, skill-based training and team-based simulation training covering scenarios they may encounter during home visits (Table 1). Teams were encouraged to work together, teach and give feedback to one another. Facilitators commented on medical content and team dynamics. Upon completion of training, each team conducted fortnightly visits over six months to one or two patients. At the midpoint and nearing the conclusion of the program, healthcare undergraduates attended multidisciplinary meetings (MDM) chaired by healthcare professionals from KTPH and staff from NWCDC. Healthcare undergraduates were expected to assess the patients' needs, present and execute a management plan under the guidance of the healthcare professionals. Each six-month visitation period is concluded with reflections on the healthcare undergraduates' learning journey. 
Table 1

Sample scenarios for training interprofessional teams

\begin{tabular}{|c|c|c|}
\hline S/No & $\begin{array}{l}\text { Learning } \\
\text { Objectives. At the } \\
\text { end of the } \\
\text { exercise, the } \\
\text { team will be } \\
\text { expected to be } \\
\text { able to: }\end{array}$ & Scenario Description \\
\hline 1. & $\begin{array}{l}\text { - Identify the } \\
\text { normal ranges } \\
\text { for vital signs. } \\
\text { • Learn to check } \\
\text { the vital signs of } \\
\text { a patient using } \\
\text { blood pressure } \\
\text { machine, } \\
\text { glucometer, } \\
\text { thermometer. } \\
\text { - Interpret the } \\
\text { possible causes } \\
\text { for abnormal } \\
\text { vital signs. }\end{array}$ & $\begin{array}{l}\text { Mdm Lee is a 53-year old Chinese lady. She has a past medical history of } \\
\text { Diabetes Mellitus Type } 2 \text { (on insulin therapy twice a day) and recurrent falls. } \\
\text { She lives with her husband and domestic helper in a three-room flat. She has } \\
\text { one daughter who is married and lives apart. } \\
\text { During the simulated home visit, the team is expected to pick up that she has a } \\
\text { fever and high capillary glucose reading. On further history taking, she will } \\
\text { reveal that she has missed her insulin injections for the last } 3 \text { days due to poor } \\
\text { appetite. She is likely to be suffering from acute diabetic crisis from both } \\
\text { omission of insulin and infection. }\end{array}$ \\
\hline 2. & $\begin{array}{l}\text { - Manage an } \\
\text { emergency or } \\
\text { accident that } \\
\text { may occur during } \\
\text { a home visit by } \\
\text { assessing the } \\
\text { airway, breathing, } \\
\text { circulation. } \\
\text { - Communicate } \\
\text { with the nurse in- } \\
\text { charge of the } \\
\text { patient using the } \\
\text { Situation- } \\
\text { Background- } \\
\text { Assessment- } \\
\text { Recommendation } \\
\text { (SBAR) format. } \\
\text { - Communicate } \\
\text { with the patient's } \\
\text { next-of-kin } \\
\text { regarding the } \\
\text { situation. }\end{array}$ & $\begin{array}{l}\text { Mdm Tan is a 60-year old Chinese lady. She has a past medical history of } \\
\text { Diabetes Mellitus Type } 2 \text { (on insulin therapy twice a day) and recurrent falls. } \\
\text { She lives with her domestic helper in a two-room flat. She has one daughter } \\
\text { who is married and lives apart. } \\
\text { Your team is visiting her for the second time. Only the domestic helper is at } \\
\text { home with Mdm Tan. You were seated in the living room while she went to the } \\
\text { kitchen to get you some drinks. Suddenly, you heard a shout. You rushed to the } \\
\text { living room and found Mdm Tan on the floor. She had slipped and fell. } \\
\text { On examination, you noted her right leg is externally rotated (likely a right hip } \\
\text { fracture). }\end{array}$ \\
\hline
\end{tabular}




\begin{tabular}{|c|c|c|}
\hline S/No & $\begin{array}{l}\text { Learning } \\
\text { Objectives. At the } \\
\text { end of the } \\
\text { exercise, the } \\
\text { team will be } \\
\text { expected to be } \\
\text { able to: }\end{array}$ & Scenario Description \\
\hline 3. & $\begin{array}{l}\text { - Identify that the } \\
\text { patient's blood } \\
\text { pressure and } \\
\text { capillary glucose } \\
\text { values are not } \\
\text { within the ideal } \\
\text { range. } \\
\text { - Identify reasons } \\
\text { for non- } \\
\text { compliance to } \\
\text { medications. } \\
\text { (financial } \\
\text { reasons, poor } \\
\text { understanding, } \\
\text { lack of perceived } \\
\text { benefit, etc) } \\
\text { - Counsel patient } \\
\text { and patient's } \\
\text { caregiver on the } \\
\text { importance of } \\
\text { adhering to } \\
\text { medications. } \\
\text { - Identify wrong } \\
\text { insulin } \\
\text { administration } \\
\text { technique. } \\
\text { - Teach patient } \\
\text { and patient's } \\
\text { caregiver the } \\
\text { correct insulin } \\
\text { administration } \\
\text { technique. }\end{array}$ & $\begin{array}{l}\text { Mr Ong is a } 70 \text {-year old Chinese man. He has the following past medical } \\
\text { history: Diabetes mellitus type 2, hypertension, hyperlipidemia, left middle } \\
\text { cerebral artery ischemic stroke. He is on the following medications: Insulin } \\
\text { (Glargine } 18 \mathrm{U} \text { in morning / } 10 \mathrm{U} \text { in night and Aspart } 8 \mathrm{U} \text { before every meal) } \\
\text { Enalapril } 20 \mathrm{mg} \mathrm{OM} \\
\text { Carvedilol } 6.25 \mathrm{mg} \mathrm{BD} \\
\text { Atorvastatin } 40 \mathrm{mg} \text { ON } \\
\text { Aggrenox (dipyridamole } 200 \mathrm{mg} \text {, aspirin } 25 \mathrm{mg} \text { ) } 1 \text { capsule BD } \\
\text { He is mostly bed/chair-bound and his wife is his main caregiver. } \\
\text { When you assess his blood pressure and capillary glucose levels, you noticed } \\
\text { that his blood pressure is } 160 \text { / } 100 \text { mmHg and his blood glucose levels are } \\
12 \text { mmol/H (fasting). You noticed that the insulin is expired and not kept in the } \\
\text { refrigerator. In addition, the patient has packs of medications that are expired } \\
\text { and seemed to be untouched }\end{array}$ \\
\hline
\end{tabular}




\begin{tabular}{|c|c|c|}
\hline S/No & $\begin{array}{l}\text { Learning } \\
\text { Objectives. At the } \\
\text { end of the } \\
\text { exercise, the } \\
\text { team will be } \\
\text { expected to be } \\
\text { able to: }\end{array}$ & Scenario Description \\
\hline 4. & $\begin{array}{l}\text { - Assess the } \\
\text { patient's } \\
\text { Activities of Daily } \\
\text { Living (ADL) and } \\
\text { Mobility } \\
\text { - Conduct a fall } \\
\text { risk assessment } \\
\text { - patient's } \\
\text { factors and } \\
\text { environmental } \\
\text { factor } \\
\text { - Develop a } \\
\text { comprehensive } \\
\text { plan to reduce } \\
\text { the risk of fall } \\
\text { - Identify } \\
\text { psychosocial } \\
\text { concerns that the } \\
\text { patient may have }\end{array}$ & $\begin{array}{l}\text { Mdm Ho is a } 84 \text { year old Chinese lady who lives alone after her husband } \\
\text { passed away } 3 \text { months ago due to a heart attack (he was her caregiver before } \\
\text { he passed away) and her children have moved out after they have started } \\
\text { families on their own. Her past medical problems include: Right lacunar stroke } \\
\text { with residual left sided weakness, hypertension, osteoarthritis, cataract bilateral. } \\
\text { Her medications include aspirin } 100 \text { mg OM, atorvastatin } 40 \text { mg ON, } \\
\text { hydrochlorothiazide } 25 \text { mg OM, atenolol } 50 \text { mg OM. } \\
\text { She lives alone in a 1-bedroom rental flat. } \\
\text { When you enter the home, you notice that the house is cluttered and dimly lit. } \\
\text { The toilet does not have a grab bar and requires the patient to squat. In } \\
\text { addition, there is a step the patient must cross to move from the kitchen to the } \\
\text { toilet. She has a hemiplegic gait and uses a umbrella to walk around the house. } \\
\text { When you speak to her, you find out that she has fallen four times in the last } \\
\text { month within the house. Midway through the conversation when you mention } \\
\text { about her husband, she starts crying. }\end{array}$ \\
\hline 5. & $\begin{array}{l}\text { - Learn to identify } \\
\text { how the home } \\
\text { visits and } \\
\text { interactions with } \\
\text { the older persons } \\
\text { affect the } \\
\text { secondary school } \\
\text { students. } \\
\text { - Encourage, } \\
\text { motivate and } \\
\text { lead the } \\
\text { secondary school } \\
\text { students within } \\
\text { the tea. }\end{array}$ & $\begin{array}{l}\text { John, a secondary three student, is one of the secondary school students on } \\
\text { your team. Together with the rest of your team, John has been consistent for } \\
\text { home visits. However, you have noticed a change in John's enthusiasm recently; } \\
\text { he has expressed a gradually reduced interest in taking initiatives when talking } \\
\text { to the elderly resident and stopped sharing during post-visit debriefs. } \\
\text { Furthermore, John has begun arriving late and leaving early for home visits. } \\
\text { This week, you have noticed that John will be missing the second home visit } \\
\text { consecutively. } \\
\text { When you speak to him, he revealed the following: } \\
\text { - He was made to join TriGen by his teacher. Nevertheless, he was willing to join } \\
\text { the programme as his best friend is in it too. } \\
\text { - He has been experiencing an increase in workload and stress from school } \\
\text { (Examinations are approaching and he has been scolded by his teachers and } \\
\text { parents for not faring well in recent class tests) } \\
\text { - He finds it challenging to interact with the older person as his grandparents } \\
\text { passed away when he was very young. }\end{array}$ \\
\hline
\end{tabular}

This IPE program was designed based on educational principles for adult learners outlined by Knowles et al.(19) First, the program provided the healthcare undergraduates with opportunities for experiential learning anchored in the service-learning approach. Second, the program was largely problem-based group learning with most of the training sessions being team-based and scenario-based. MDMs were also problem-based; these sessions encouraged the undergraduates to grapple with issues of their patients and brainstorm ideas to address them. Third, the service they provided in this program modeled the work they may engage in after graduation. What they learned in this program was of immediate relevance to their current study and future practice. Lastly, the program 
provided autonomy to healthcare undergraduates to direct their own learning. This program is voluntary, and most participated because they were drawn by the elements of IPE and IPC. In addition, the program allowed flexibility for further self-study of topics of interest.

TriGen was built upon the contact hypothesis, which proposes that contact between different groups can change attitudes for the better.(20) Several factors are needed to ensure the success of these contacts: equal status of participants; positive expectations; a co-operative atmosphere; successful joint work; a concern for and understanding of differences as well as similarities between different professions; the experience of working together as equals.(21)

\section{Evaluation Approach}

This study used the Kirkpatrick's framework expanded by Barr et al to evaluate the effectiveness of the program in improving healthcare undergraduates' attitudes towards IPE.(8) A multimethod approach was used for an indepth analysis of the impact of IP learning in undergraduates through the administrations of the Readiness for Interprofessional Learning Scale (RIPLS) pre- and post-intervention, and post-intervention quantitative and qualitative feedback.(22) Table 2 summarises the measures and methods used. 
Table 2

Components of Kirkpatrick/Barr et al. Evaluation Framework as Applied to TriGen

\begin{tabular}{|c|c|c|}
\hline Evaluation Level & $\begin{array}{l}\text { Methods and } \\
\text { Measures }\end{array}$ & Timeframe \\
\hline \multirow{3}{*}{$\begin{array}{l}\text { Level 1: Learners' reactions } \\
\text { Participants' views of their learning experience and satisfaction with the } \\
\text { program }\end{array}$} & $\begin{array}{l}\text { - Readiness for } \\
\text { Interprofessional }\end{array}$ & $\begin{array}{l}\text { - Pre- and } \\
\text { post- }\end{array}$ \\
\hline & Learning Scale & intervention \\
\hline & $\begin{array}{l}\text { - Qualitative } \\
\text { feedback }\end{array}$ & $\begin{array}{l}\text { - Post- } \\
\text { intervention }\end{array}$ \\
\hline \multirow[t]{2}{*}{ Level 2a: Modification of attitudes perceptions } & $\begin{array}{l}\text { - Participants' } \\
\text { self-reported } \\
\text { feedback of IPE } \\
\text { learning }\end{array}$ & $\begin{array}{l}\text { - Post- } \\
\text { intervention }\end{array}$ \\
\hline & $\begin{array}{l}\text { - Qualitative } \\
\text { feedback }\end{array}$ & $\begin{array}{l}\text { • Post- } \\
\text { intervention }\end{array}$ \\
\hline $\begin{array}{l}\text { Level 2b: Acquisition of knowledge/skills } \\
\text { Concepts, procedures, principles and skills }\end{array}$ & $\begin{array}{l}\text { - Qualitative } \\
\text { feedback }\end{array}$ & $\begin{array}{l}\text { - Post- } \\
\text { intervention }\end{array}$ \\
\hline $\begin{array}{l}\text { Level 3: Change in behavior } \\
\text { Behavioral change transferred from the learning environment to the } \\
\text { workplace prompted by modifications in attitudes or perceptions, or the } \\
\text { application of newly acquired knowledge or skills in practice }\end{array}$ & $\begin{array}{l}\text { - Qualitative } \\
\text { feedback }\end{array}$ & $\begin{array}{l}\text { - Post- } \\
\text { intervention }\end{array}$ \\
\hline \multirow[t]{2}{*}{$\begin{array}{l}\text { Level 4a: Change in organizational practice } \\
\text { Wider changes in the organization and/or delivery of care, attributable to } \\
\text { an education program }\end{array}$} & $\begin{array}{l}\text { - Emergency } \\
\text { department } \\
\text { readmission } \\
\text { rates* }\end{array}$ & $\begin{array}{l}\text { - Pre- and } \\
\text { post- } \\
\text { intervention }\end{array}$ \\
\hline & $\begin{array}{l}\text { - Hospital } \\
\text { readmission } \\
\text { rates* }\end{array}$ & $\begin{array}{l}\text { - Pre- and } \\
\text { post- } \\
\text { intervention }\end{array}$ \\
\hline $\begin{array}{l}\text { Level 4b: Benefits to patients/service users } \\
\text { Improvements in the health and wellbeing of patients/service users as a } \\
\text { direct result of an educational program }\end{array}$ & $\begin{array}{l}\text { - Patients' } \\
\text { satisfaction* }\end{array}$ & $\begin{array}{l}\text { - Post- } \\
\text { intervention }\end{array}$ \\
\hline
\end{tabular}

\section{Quantitative Measures}

The RIPLS was administered pre- and post-intervention. The RIPLS was formulated as a 19-item questionnaire comprising 3 subscales (Teamwork and Collaboration; Professional Identity; and Roles and Responsibilities), wherein some items are negatively coded.(23) Higher RIPLS scores imply greater readiness for IP learning. The RIPLS was chosen as it has been shown to be a valid and useful tool for measuring attitudes towards IPE especially in the context of healthcare students, $(24,25)$ and has been validated for use in the local context.(26)

\section{Statistical Analysis}

The Shapiro-Wilk test was used to assess if the data followed a normal distribution.(27) A paired $t$-test comparing baseline and post-intervention responses was computed for each survey item to determine significant attitude 
differences ( $p \leq 0.05)$. One-way analysis of variance (ANOVA) was performed to assess for demographic factors that correlated with pre-intervention and magnitude of change in RIPLS scores. If one-way ANOVA demonstrated an overall difference between groups, post-hoc Tukey's honestly significant difference (Tukey's HSD) test was performed. Pearson correlation was used to identify correlations in baseline and post-intervention scores. For all statistical analyses, the Statistical Package for Social Sciences (SPSS, Version 23.0, Chicago, Illinois) was used.

\section{Internal Consistency}

Cronbach's alpha was used to assess internal consistency of RIPLS, $a=0.848$ for RIPLS total score.

\section{Qualitative Measures}

Post-intervention qualitative feedback regarding their learning experiences was collected through online surveys. Questions include: What did you learn about interprofessional collaboration? What are your learning points after completing the project? Would you recommend this project to your peers, what are your reasons? Thematic analysis was performed on the students' qualitative descriptions of their learning experiences.

\section{Thematic Analysis}

A grounded theory approach was used.(28) Constant comparison analysis was used to identify patterns in participants' responses and develop a coding schema. Two coders (KYYN and GYCL) independently identified major themes from text within all transcripts, with reference to the research questions. Both coders discussed and resolved any disagreements. A common coding schema was generated and applied to all the transcripts.

\section{Ethics}

Ethical approval was obtained from the NUS institutional review board (B-15-272) to evaluate the learning outcomes of the program. Study participation was entirely voluntary and anonymous. Informed consent was taken from participants before the commencement of data collection. There were no incentives provided to study participants.

\section{Results}

226 healthcare undergraduates participated in TriGen from 2015 to 2018, with a response rate of $80.1 \%$. Table 3 describes the participants' demographics. During this period, 106 patients and 359 secondary school students also participated in TriGen. 
Table 3

Demographic profile of students who participated in the TriGen Programme from 2015-2017

\begin{tabular}{|c|c|}
\hline & Healthcare undergraduates \\
\hline Total number of participants & 226 \\
\hline Number of respondents & 181 \\
\hline Response rate & $80.1 \%$ \\
\hline Median Age (Range) & $21(18-41)$ \\
\hline \multicolumn{2}{|l|}{ Gender } \\
\hline Male & $68(37.6 \%)$ \\
\hline Female & $113(62.4 \%)$ \\
\hline \multicolumn{2}{|l|}{ Faculty/School } \\
\hline Medicine & $57(31.5 \%)$ \\
\hline Nursing & $23(12.7 \%)$ \\
\hline Pharmacy & $76(42.0 \%)$ \\
\hline Social Work & $20(11.0 \%)$ \\
\hline Physiotherapy and Occupational Therapy & $5(2.8 \%)$ \\
\hline \multicolumn{2}{|l|}{ Year of study } \\
\hline Year 1 & $72(39.8 \%)$ \\
\hline Year 2 & $41(22.7 \%)$ \\
\hline Year 3 & $52(28.7 \%)$ \\
\hline Year 4 & $14(7.7 \%)$ \\
\hline Year 5 & $2(1.7 \%)$ \\
\hline \multicolumn{2}{|l|}{ Living with grandparents } \\
\hline Yes & $25(13.8 \%)$ \\
\hline No & $156(86.2 \%)$ \\
\hline \multicolumn{2}{|l|}{ Involved in volunteer work with older person(s) } \\
\hline Yes & $131(72.4 \%)$ \\
\hline No & $50(27.6 \%)$ \\
\hline \multicolumn{2}{|l|}{ Previous IP education activities } \\
\hline Yes & $117(64.6 \%)$ \\
\hline No & $64(35.4 \%)$ \\
\hline
\end{tabular}




\section{Baseline RIPLS Score}

At baseline, there was a significant difference between different faculties for total RIPLS score at baseline $(p=$ 0.004). Pharmacy students had lower baseline total RIPLS scores as compared to medical students (mean difference $1.92,0.103-3.75, p=0.033$ ) and therapy students (mean difference $5.35,0.55-10.2, p=0.021$ ). There was no difference in total RIPLS score between different gender, year of study, previous exposure to IPE projects and current or past participation in activities outside of the faculty. (Table 4) 
Table 4

RIPLS (Total score)

\begin{tabular}{|c|c|c|c|c|c|c|c|}
\hline Group & No. & $\begin{array}{l}\text { Pre- } \\
\text { intervention } \\
\text { group score } \\
\text { (mean, } \\
95 \% \mathrm{Cl} \text { ) }\end{array}$ & $\begin{array}{l}\text { P-value } \\
\text { (comparison } \\
\text { between } \\
\text { groups for } \\
\text { pre- } \\
\text { intervention } \\
\text { score) }\end{array}$ & $\begin{array}{l}\text { Post- } \\
\text { intervention } \\
\text { group score } \\
\text { (mean, } \\
95 \% \mathrm{Cl} \text { ) }\end{array}$ & $\begin{array}{l}\text { Mean } \\
\text { difference } \\
\text { between } \\
\text { post minus } \\
\text { pre } \\
\text { intervention } \\
\text { scores } \\
\text { (mean, } \\
95 \% \mathrm{Cl} \text { ) }\end{array}$ & $\begin{array}{l}\text { P-value for } \\
\text { mean } \\
\text { difference } \\
\text { between } \\
\text { post minus } \\
\text { pre } \\
\text { intervention } \\
\text { scores }\end{array}$ & $\begin{array}{l}\text { P-value for } \\
\text { comparison } \\
\text { between } \\
\text { pre and } \\
\text { post } \\
\text { intervention } \\
\text { group } \\
\text { scores }\end{array}$ \\
\hline $\begin{array}{l}\text { All } \\
\text { University } \\
\text { Students }\end{array}$ & 175 & $\begin{array}{l}80.3 \text { (79.2- } \\
81.4\end{array}$ & NA & $\begin{array}{l}79.0(77.8- \\
80.3)\end{array}$ & $\begin{array}{l}-1.3(-2.5- \\
-0.089)\end{array}$ & 0.035 & \\
\hline Gender & & & 0.344 & & & & 0.256 \\
\hline Male & 65 & $\begin{array}{l}80.6(78.6- \\
82.5)\end{array}$ & & $\begin{array}{l}78.4(76.2- \\
80.6)\end{array}$ & $\begin{array}{l}-2.2(-4.14 \\
--0.23)\end{array}$ & 0.029 & \\
\hline Female & 110 & $\begin{array}{l}80.2(78.8- \\
81.5)\end{array}$ & & $\begin{array}{l}79.4(77.8- \\
80.9)\end{array}$ & $\begin{array}{l}-0.76(- \\
2.3-0.77)\end{array}$ & 0.330 & \\
\hline $\begin{array}{l}\text { Year of } \\
\text { Study }\end{array}$ & & & 0.240 & & & & 0.054 \\
\hline $\begin{array}{l}\text { Year } 1 \\
\text { and } 2\end{array}$ & 109 & $\begin{array}{l}80.9(79.5- \\
82.3)\end{array}$ & & $\begin{array}{l}78.7(77.0- \\
80.4)\end{array}$ & $\begin{array}{l}-2.19(- \\
3.86- \\
-0.53)\end{array}$ & 0.010 & \\
\hline $\begin{array}{l}\text { Year } 3 \text { to } \\
5\end{array}$ & 66 & $\begin{array}{l}79.3(77.6- \\
81.0)\end{array}$ & & $\begin{array}{l}79.6(77.6- \\
81.5)\end{array}$ & $\begin{array}{l}0.21 \\
(-1.39- \\
1.79)\end{array}$ & 0.789 & \\
\hline Faculty & & & 0.004 & & & & 0.658 \\
\hline Medical & 57 & $\begin{array}{l}82.1(80.4- \\
83.8)\end{array}$ & & $\begin{array}{l}79.7 \text { (77.4- } \\
81.9)\end{array}$ & $\begin{array}{l}-2.44(- \\
4.8-- \\
0.086)\end{array}$ & 0.042 & \\
\hline Nursing & 23 & $\begin{array}{l}82.4(78.8- \\
86.0)\end{array}$ & & $\begin{array}{l}81.1(77.6- \\
84.6)\end{array}$ & $\begin{array}{l}-1.35(- \\
5.46-2.76)\end{array}$ & 0.504 & \\
\hline Pharmacy & 71 & $\begin{array}{l}78.0(76.4- \\
79.7)\end{array}$ & & $\begin{array}{l}77.8(75.8- \\
79.8)\end{array}$ & $\begin{array}{l}9.18(3.99- \\
14.36)\end{array}$ & 0.762 & \\
\hline $\begin{array}{l}\text { Social } \\
\text { Work }\end{array}$ & 19 & $\begin{array}{l}78.2(75.0- \\
81.3)\end{array}$ & & $\begin{array}{l}76.8(73.8- \\
79.9)\end{array}$ & $\begin{array}{l}-1.32(- \\
5.00-2.37)\end{array}$ & 0.463 & \\
\hline Therapist & 5 & $\begin{array}{l}90.4(87.2- \\
93.6)\end{array}$ & & $\begin{array}{l}88.0(82.6- \\
93.4)\end{array}$ & $\begin{array}{l}-2.4(- \\
10.00- \\
5.18)\end{array}$ & 0.429 & \\
\hline $\begin{array}{l}\text { Previous } \\
\text { IPE } \\
\text { projects }\end{array}$ & & & 0.525 & & & & 0.740 \\
\hline Yes & 112 & $\begin{array}{l}80.5(79.1- \\
82.0)\end{array}$ & & $\begin{array}{l}79.1(77.5- \\
80.8)\end{array}$ & $\begin{array}{l}-1.44(- \\
3.04-0.17)\end{array}$ & 0.079 & \\
\hline
\end{tabular}




\begin{tabular}{|c|c|c|c|c|c|c|c|}
\hline Group & No. & $\begin{array}{l}\text { Pre- } \\
\text { intervention } \\
\text { group score } \\
\text { (mean, } \\
95 \% \mathrm{Cl} \text { ) }\end{array}$ & $\begin{array}{l}\text { P-value } \\
\text { (comparison } \\
\text { between } \\
\text { groups for } \\
\text { pre- } \\
\text { intervention } \\
\text { score) }\end{array}$ & $\begin{array}{l}\text { Post- } \\
\text { intervention } \\
\text { group score } \\
\text { (mean, } \\
95 \% \mathrm{Cl} \text { ) }\end{array}$ & $\begin{array}{l}\text { Mean } \\
\text { difference } \\
\text { between } \\
\text { post minus } \\
\text { pre } \\
\text { intervention } \\
\text { scores } \\
\text { (mean, } \\
95 \% \mathrm{Cl} \text { ) }\end{array}$ & $\begin{array}{l}\text { P-value for } \\
\text { mean } \\
\text { difference } \\
\text { between } \\
\text { post minus } \\
\text { pre } \\
\text { intervention } \\
\text { scores }\end{array}$ & $\begin{array}{l}\text { P-value for } \\
\text { comparison } \\
\text { between } \\
\text { pre and } \\
\text { post } \\
\text { intervention } \\
\text { group } \\
\text { scores }\end{array}$ \\
\hline No & 63 & $\begin{array}{l}79.9(78.1- \\
81.6)\end{array}$ & & $\begin{array}{l}78.9(76.9- \\
80.8)\end{array}$ & $\begin{array}{l}-1.01(- \\
2.78-0.75)\end{array}$ & 0.254 & \\
\hline $\begin{array}{l}\text { Activities } \\
\text { Outside } \\
\text { Faculty }\end{array}$ & & & 0.120 & & & & 0.125 \\
\hline Yes & 40 & $\begin{array}{l}79.4(77.1- \\
81.6)\end{array}$ & & $\begin{array}{l}79.8(77.3- \\
82.3)\end{array}$ & $\begin{array}{l}0.43(- \\
1.70-2.54)\end{array}$ & 0.687 & \\
\hline No & 135 & $\begin{array}{l}80.6(79.3- \\
81.9)\end{array}$ & & $\begin{array}{l}78.8(77.3- \\
80.3)\end{array}$ & $\begin{array}{l}-1.79 \\
(-3.21- \\
0.37)\end{array}$ & 0.014 & \\
\hline
\end{tabular}

For the "teamwork" subscale, there was a significant difference between early (Year 1-2) vs. later (Year 3-5) years of study with Year 1-2 undergraduates having a higher baseline score of $40.9(40.1-41.6)$ versus Year 3-5 undergraduates with a score of $39.5(38.6-40.4)(p=0.034)$. There was also a significant difference amongst faculties $(p=0.019)$, with therapy students scoring highest at $44.6(44.1-45.1)$ compared to medical students at 41.2 (40.2-42.2), nursing students at 41.4 (39.7-43.0), pharmacy students at 39.3 (38.4-40.1) and social work students at 39.5 (37.3-41.6). Therapy students had a significantly higher baseline score compared to pharmacy students $(p=0.043)$. There was no difference in "teamwork" subscale score between genders, previous exposure to IPE projects and current or past participation in activities outside of the faculty. (Table 5) 
Table 5

RIPLS (Teamwork subscale)

\begin{tabular}{|c|c|c|c|c|c|c|c|}
\hline Group & No. & $\begin{array}{l}\text { Pre- } \\
\text { intervention } \\
\text { group score } \\
\text { (mean, } \\
95 \% \mathrm{Cl} \text { ) }\end{array}$ & $\begin{array}{l}\text { P-value } \\
\text { (comparison } \\
\text { between } \\
\text { groups for } \\
\text { pre- } \\
\text { intervention } \\
\text { score) }\end{array}$ & $\begin{array}{l}\text { Post- } \\
\text { intervention } \\
\text { group score } \\
\text { (mean, } \\
95 \% \mathrm{Cl})\end{array}$ & $\begin{array}{l}\text { Mean } \\
\text { difference } \\
\text { between } \\
\text { post minus } \\
\text { pre } \\
\text { intervention } \\
\text { scores } \\
\text { (mean, } \\
95 \% \mathrm{Cl} \text { ) }\end{array}$ & $\begin{array}{l}\text { P-value for } \\
\text { mean } \\
\text { difference } \\
\text { between } \\
\text { post minus } \\
\text { pre } \\
\text { intervention } \\
\text { scores }\end{array}$ & $\begin{array}{l}\text { P-value for } \\
\text { comparison } \\
\text { between } \\
\text { pre and } \\
\text { post } \\
\text { intervention } \\
\text { group } \\
\text { scores }\end{array}$ \\
\hline $\begin{array}{l}\text { All } \\
\text { University } \\
\text { Students }\end{array}$ & 175 & $\begin{array}{l}40.3(39.7- \\
40.9)\end{array}$ & & $\begin{array}{l}40.0(39.4- \\
40.6)\end{array}$ & $\begin{array}{l}-0.30(- \\
0.90-0.29)\end{array}$ & 0.317 & \\
\hline Gender & & & 0.185 & & & & 0.236 \\
\hline Male & 65 & $\begin{array}{l}40.7(39.7- \\
41.7)\end{array}$ & & $\begin{array}{l}39.9(38.8- \\
41.0)\end{array}$ & $\begin{array}{l}-0.77(- \\
1.67-0.13)\end{array}$ & 0.092 & \\
\hline Female & 110 & $\begin{array}{l}40.2(39.4- \\
40.9)\end{array}$ & & $\begin{array}{l}40.1(39.4- \\
40.8)\end{array}$ & $\begin{array}{l}-0.027(- \\
0.82-0.77)\end{array}$ & 0.946 & \\
\hline $\begin{array}{l}\text { Year of } \\
\text { Study }\end{array}$ & & & 0.034 & & & & 0.101 \\
\hline $\begin{array}{l}\text { Year } 1 \\
\text { and } 2\end{array}$ & 109 & $\begin{array}{l}40.9(40.1- \\
41.6)\end{array}$ & & $\begin{array}{l}40.2(39.4- \\
40.9)\end{array}$ & $\begin{array}{l}-0.69(- \\
1.45- \\
0.074)\end{array}$ & 0.076 & \\
\hline $\begin{array}{l}\text { Year } 3 \text { to } \\
5\end{array}$ & 66 & $\begin{array}{l}39.5(38.6- \\
40.4)\end{array}$ & & $\begin{array}{l}39.8(38.8- \\
40.9)\end{array}$ & $\begin{array}{l}0.33(- \\
0.63-1.30)\end{array}$ & 0.491 & \\
\hline Faculty & & & 0.019 & & & & 0.194 \\
\hline Medical & 57 & $\begin{array}{l}41.2(40.2- \\
42.2)\end{array}$ & & $\begin{array}{l}40.5(39.5- \\
41.5)\end{array}$ & $\begin{array}{l}-0.67(- \\
1.77-0.43)\end{array}$ & 0.229 & \\
\hline Nursing & 23 & $\begin{array}{l}41.4(39.7- \\
43.0)\end{array}$ & & $\begin{array}{l}39.8(38.2- \\
41.5)\end{array}$ & $\begin{array}{l}-15.2(- \\
3.38-0.34)\end{array}$ & 0.103 & \\
\hline Pharmacy & 71 & $\begin{array}{l}39.3(38.4- \\
40.1)\end{array}$ & $\begin{array}{l}\text { Therapist } \\
(0.043)\end{array}$ & $\begin{array}{l}39.8(38.8- \\
40.8)\end{array}$ & $\begin{array}{l}0.52(- \\
0.34-1.38)\end{array}$ & 0.230 & \\
\hline $\begin{array}{l}\text { Social } \\
\text { Work }\end{array}$ & 19 & $\begin{array}{l}39.5(37.3- \\
41.6)\end{array}$ & & $\begin{array}{l}39.0(37.4- \\
40.5)\end{array}$ & $\begin{array}{l}-0.53(- \\
2.68-1.63)\end{array}$ & 0.615 & \\
\hline Therapist & 5 & $\begin{array}{l}44.6(44.1- \\
45.1)\end{array}$ & & $\begin{array}{l}43.2(41.0- \\
45.4)\end{array}$ & $\begin{array}{l}-1.40(- \\
5.00-2.18)\end{array}$ & 0.338 & \\
\hline $\begin{array}{l}\text { Previous } \\
\text { IPE } \\
\text { projects }\end{array}$ & & & 0.820 & & & & 0.909 \\
\hline Yes & 112 & $\begin{array}{l}40.5(39.8- \\
41.2)\end{array}$ & & $\begin{array}{l}40.3(39.5- \\
41.0)\end{array}$ & $\begin{array}{l}-0.28(- \\
1.02-0.47)\end{array}$ & 0.464 & \\
\hline No & 63 & $\begin{array}{l}40.0(39.0- \\
41.1)\end{array}$ & & $\begin{array}{l}39.7(38.7- \\
40.6)\end{array}$ & $\begin{array}{l}-0.35 \\
(-1.37- \\
0.67)\end{array}$ & 0.495 & \\
\hline
\end{tabular}




\begin{tabular}{|c|c|c|c|c|c|c|c|}
\hline Group & No. & $\begin{array}{l}\text { Pre- } \\
\text { intervention } \\
\text { group score } \\
\text { (mean, } \\
95 \% \mathrm{Cl} \text { ) }\end{array}$ & $\begin{array}{l}\text { P-value } \\
\text { (comparison } \\
\text { between } \\
\text { groups for } \\
\text { pre- } \\
\text { intervention } \\
\text { score) }\end{array}$ & $\begin{array}{l}\text { Post- } \\
\text { intervention } \\
\text { group score } \\
\text { (mean, } \\
95 \% \mathrm{Cl} \text { ) }\end{array}$ & $\begin{array}{l}\text { Mean } \\
\text { difference } \\
\text { between } \\
\text { post minus } \\
\text { pre } \\
\text { intervention } \\
\text { scores } \\
\text { (mean, } \\
95 \% \mathrm{Cl})\end{array}$ & $\begin{array}{l}\text { P-value for } \\
\text { mean } \\
\text { difference } \\
\text { between } \\
\text { post minus } \\
\text { pre } \\
\text { intervention } \\
\text { scores }\end{array}$ & $\begin{array}{l}\text { P-value for } \\
\text { comparison } \\
\text { between } \\
\text { pre and } \\
\text { post } \\
\text { intervention } \\
\text { group } \\
\text { scores }\end{array}$ \\
\hline $\begin{array}{l}\text { Activities } \\
\text { Outside } \\
\text { Faculty }\end{array}$ & & & 0.376 & & & & 0.095 \\
\hline Yes & 40 & $\begin{array}{l}40.1(38.9- \\
41.3)\end{array}$ & & $\begin{array}{l}40.8(39.6- \\
41.9)\end{array}$ & $\begin{array}{l}0.623(- \\
0.50-1.75)\end{array}$ & 0.269 & \\
\hline No & 135 & $\begin{array}{l}40.4(39.7- \\
41.1)\end{array}$ & & $\begin{array}{l}39.8(39.1- \\
40.5)\end{array}$ & $\begin{array}{l}-0.58(- \\
1.28-0.12)\end{array}$ & 0.103 & \\
\hline
\end{tabular}

For the "professional identity" subscale, there was no difference in the score between genders, faculty, year of study, previous exposure to IPE projects and current or past participation in activities outside of the faculty. (Table 6) 
Table 6

RIPLS (Professional identity)

\begin{tabular}{|c|c|c|c|c|c|c|c|}
\hline Group & No. & $\begin{array}{l}\text { Pre- } \\
\text { intervention } \\
\text { group score } \\
\text { (mean, } \\
95 \% \mathrm{Cl} \text { ) }\end{array}$ & $\begin{array}{l}\text { P-value } \\
\text { (comparison } \\
\text { between } \\
\text { groups for } \\
\text { pre- } \\
\text { intervention } \\
\text { score) }\end{array}$ & $\begin{array}{l}\text { Post- } \\
\text { intervention } \\
\text { group score } \\
\text { (mean, } \\
95 \% \mathrm{Cl} \text { ) }\end{array}$ & $\begin{array}{l}\text { Mean } \\
\text { difference } \\
\text { between } \\
\text { post minus } \\
\text { pre } \\
\text { intervention } \\
\text { scores } \\
\text { (mean, } \\
95 \% \mathrm{Cl} \text { ) }\end{array}$ & $\begin{array}{l}\text { P-value for } \\
\text { mean } \\
\text { difference } \\
\text { between } \\
\text { post minus } \\
\text { pre } \\
\text { intervention } \\
\text { scores }\end{array}$ & $\begin{array}{l}\text { P-value for } \\
\text { comparison } \\
\text { between } \\
\text { pre and } \\
\text { post } \\
\text { intervention } \\
\text { group } \\
\text { scores }\end{array}$ \\
\hline $\begin{array}{l}\text { All } \\
\text { University } \\
\text { Students }\end{array}$ & 175 & $\begin{array}{l}30.2(29.7- \\
30.8)\end{array}$ & & $\begin{array}{l}28.8(28.1- \\
29.5)\end{array}$ & $\begin{array}{l}-1.4(-2.1 \\
\text { to }-0.77)\end{array}$ & $<0.001$ & \\
\hline Gender & & & 0.936 & & & & 0.928 \\
\hline Male & 65 & $\begin{array}{l}30.0(29.1- \\
30.9)\end{array}$ & & $\begin{array}{l}28.6(27.5- \\
29.8)\end{array}$ & $\begin{array}{l}-1.4(-2.6 \\
--0.23)\end{array}$ & 0.020 & \\
\hline Female & & $\begin{array}{l}30.4(29.7- \\
31.0)\end{array}$ & & $\begin{array}{l}28.9(28.0- \\
29.8)\end{array}$ & $\begin{array}{l}-1.46(- \\
2.29- \\
-0.64)\end{array}$ & 0.001 & \\
\hline $\begin{array}{l}\text { Year of } \\
\text { Study }\end{array}$ & & & 0.393 & & & & 0.077 \\
\hline $\begin{array}{l}\text { Year } 1 \\
\text { and } 2\end{array}$ & 109 & $\begin{array}{l}30.4(29.8- \\
31.1)\end{array}$ & & $\begin{array}{l}28.5(27.6- \\
29.5)\end{array}$ & $\begin{array}{l}-1.91(- \\
2.87-- \\
0.94)\end{array}$ & $<0.001$ & \\
\hline $\begin{array}{l}\text { Year } 3 \text { to } \\
5\end{array}$ & 66 & $\begin{array}{l}29.9(29.1- \\
30.7)\end{array}$ & & $\begin{array}{l}29.2(28.2- \\
30.2)\end{array}$ & $\begin{array}{l}-0.67(- \\
1.46-0.13)\end{array}$ & 0.098 & \\
\hline Faculty & & & 0.056 & & & & 0.466 \\
\hline Medical & 57 & $\begin{array}{l}30.9(30.1- \\
31.7)\end{array}$ & & $\begin{array}{l}28.7(27.4- \\
30.1)\end{array}$ & $\begin{array}{l}-2.18(- \\
3.56-- \\
0.79)\end{array}$ & 0.003 & \\
\hline Nursing & 23 & $\begin{array}{l}30.7(28.9- \\
32.4)\end{array}$ & & $\begin{array}{l}30.0(28.5- \\
31.6)\end{array}$ & $\begin{array}{l}-0.61(- \\
2.50-1.28)\end{array}$ & 0.512 & \\
\hline Pharmacy & 71 & $\begin{array}{l}29.2(28.4- \\
30.0)\end{array}$ & & $\begin{array}{l}28.3(27.2- \\
29.4\end{array}$ & $\begin{array}{l}-0.93(- \\
1.89- \\
0.028)\end{array}$ & 0.057 & \\
\hline $\begin{array}{l}\text { Social } \\
\text { Work }\end{array}$ & 19 & $\begin{array}{l}30.6(29.3- \\
32.0)\end{array}$ & & $\begin{array}{l}28.6(26.8- \\
30.3)\end{array}$ & $\begin{array}{l}-2.05(- \\
4.14- \\
0.033)\end{array}$ & 0.053 & \\
\hline Therapist & 5 & $\begin{array}{l}33.6(32.6- \\
34.6)\end{array}$ & & $\begin{array}{l}31.8(29.0- \\
34.6)\end{array}$ & $\begin{array}{l}-1.80 \\
(-5.13- \\
1.53)\end{array}$ & 0.208 & \\
\hline $\begin{array}{l}\text { Previous } \\
\text { IPE } \\
\text { projects }\end{array}$ & & & 0.591 & & & & 0.788 \\
\hline
\end{tabular}




\begin{tabular}{|c|c|c|c|c|c|c|c|}
\hline Group & No. & $\begin{array}{l}\text { Pre- } \\
\text { intervention } \\
\text { group score } \\
\text { (mean, } \\
95 \% \mathrm{Cl})\end{array}$ & $\begin{array}{l}\text { P-value } \\
\text { (comparison } \\
\text { between } \\
\text { groups for } \\
\text { pre- } \\
\text { intervention } \\
\text { score) }\end{array}$ & $\begin{array}{l}\text { Post- } \\
\text { intervention } \\
\text { group score } \\
\text { (mean, } \\
95 \% \mathrm{Cl} \text { ) }\end{array}$ & $\begin{array}{l}\text { Mean } \\
\text { difference } \\
\text { between } \\
\text { post minus } \\
\text { pre } \\
\text { intervention } \\
\text { scores } \\
\text { (mean, } \\
95 \% \mathrm{Cl} \text { ) }\end{array}$ & $\begin{array}{l}\text { P-value for } \\
\text { mean } \\
\text { difference } \\
\text { between } \\
\text { post minus } \\
\text { pre } \\
\text { intervention } \\
\text { scores }\end{array}$ & $\begin{array}{l}\text { P-value for } \\
\text { comparison } \\
\text { between } \\
\text { pre and } \\
\text { post } \\
\text { intervention } \\
\text { group } \\
\text { scores }\end{array}$ \\
\hline Yes & 112 & $\begin{array}{l}30.1(29.4- \\
30.8)\end{array}$ & & $\begin{array}{l}28.6(27.7- \\
29.5)\end{array}$ & $\begin{array}{l}-1.51 \\
(-2.42- \\
0.59)\end{array}$ & 0.001 & \\
\hline No & 63 & $\begin{array}{l}30.4(29.6- \\
31.2)\end{array}$ & & $\begin{array}{l}29.1(28.1- \\
30.1)\end{array}$ & $\begin{array}{l}-1.32(- \\
2.26-0.37)\end{array}$ & 0.007 & \\
\hline $\begin{array}{l}\text { Activities } \\
\text { Outside } \\
\text { Faculty }\end{array}$ & & & 0.129 & & & & 0.167 \\
\hline Yes & 40 & $\begin{array}{l}29.9(28.7- \\
31.1)\end{array}$ & & $\begin{array}{l}29.3(28.0- \\
30.7)\end{array}$ & $\begin{array}{l}-0.58(- \\
1.84-0.69)\end{array}$ & 0.363 & \\
\hline No & 135 & $\begin{array}{l}30.3(29.8- \\
30.9)\end{array}$ & & $\begin{array}{l}28.6(27.8- \\
29.4)\end{array}$ & $\begin{array}{l}-1.70(- \\
2.49- \\
-0.91)\end{array}$ & $<0.001$ & \\
\hline
\end{tabular}

For the "roles and responsibility" subscale, there was a significant difference between undergraduates of different faulty $(p<0.001)$. Social work undergraduates had a lower score $8.1(7.3-8.8)$ compared to pharmacy undergraduates $9.6(9.2-10.0)$ medical undergraduates 10.0 (9.5-10.5), nursing undergraduates 10.4 (9.7-11.2). Therapists had the highest score of 12.2 (9.9-14.5). (Table 7) 
Table 7

RIPLS (Roles and responsibility subscale)

\begin{tabular}{|c|c|c|c|c|c|c|c|}
\hline Group & No. & $\begin{array}{l}\text { Pre- } \\
\text { intervention } \\
\text { group score } \\
\text { (mean, } \\
95 \% \mathrm{Cl} \text { ) }\end{array}$ & $\begin{array}{l}\text { P-value } \\
\text { (comparison } \\
\text { between } \\
\text { groups for } \\
\text { pre- } \\
\text { intervention } \\
\text { score) }\end{array}$ & $\begin{array}{l}\text { Post- } \\
\text { intervention } \\
\text { group score } \\
\text { (mean, } \\
95 \% \mathrm{Cl} \text { ) }\end{array}$ & $\begin{array}{l}\text { Mean } \\
\text { difference } \\
\text { between } \\
\text { post minus } \\
\text { pre } \\
\text { intervention } \\
\text { scores } \\
\text { (mean, } \\
95 \% \mathrm{Cl} \text { ) }\end{array}$ & $\begin{array}{l}\text { P-value for } \\
\text { mean } \\
\text { difference } \\
\text { between } \\
\text { post minus } \\
\text { pre } \\
\text { intervention } \\
\text { scores }\end{array}$ & $\begin{array}{l}\text { P-value for } \\
\text { comparison } \\
\text { between } \\
\text { pre and } \\
\text { post } \\
\text { intervention } \\
\text { group } \\
\text { scores }\end{array}$ \\
\hline $\begin{array}{l}\text { All } \\
\text { University } \\
\text { Students }\end{array}$ & 175 & $\begin{array}{l}9.7(9.4- \\
10.0)\end{array}$ & & $\begin{array}{l}10.2(9.9- \\
10.5)\end{array}$ & $\begin{array}{l}0.46(0.14- \\
0.77)\end{array}$ & 0.005 & \\
\hline Gender & & & 0.455 & & & & 0.502 \\
\hline Male & 65 & $\begin{array}{l}9.9(9.4- \\
10.4)\end{array}$ & & $\begin{array}{l}9.9(9.3- \\
10.4)\end{array}$ & $\begin{array}{l}-0.15(- \\
0.49-0.45)\end{array}$ & 0.948 & \\
\hline Female & 110 & $\begin{array}{l}9.7(9.3- \\
10.0)\end{array}$ & & $\begin{array}{l}10.4(10.0- \\
10.8)\end{array}$ & $\begin{array}{l}0.74(0.32- \\
1.15)\end{array}$ & 0.001 & \\
\hline $\begin{array}{l}\text { Year of } \\
\text { Study }\end{array}$ & & & 0.150 & & & & 0.693 \\
\hline $\begin{array}{l}\text { Year } 1 \\
\text { and } 2\end{array}$ & 109 & $\begin{array}{l}9.6(9.2- \\
10.0)\end{array}$ & & $\begin{array}{l}10.0(9.6- \\
10.4)\end{array}$ & $\begin{array}{l}0.40(- \\
0.012- \\
0.82)\end{array}$ & 0.057 & \\
\hline $\begin{array}{l}\text { Year } 3 \text { to } \\
5\end{array}$ & 66 & $\begin{array}{l}10.0(9.6- \\
10.4)\end{array}$ & & $\begin{array}{l}10.5 \text { (10.1- } \\
10.9)\end{array}$ & $\begin{array}{l}0.55 \\
(0.057- \\
1.03)\end{array}$ & 0.029 & \\
\hline Faculty & & & $<0.001$ & & & & 0.576 \\
\hline Medical & 57 & $\begin{array}{l}10.0(9.5- \\
10.5)\end{array}$ & $\begin{array}{l}\text { Social work } \\
(0.002)\end{array}$ & $\begin{array}{l}10.4(9.9- \\
10.9)\end{array}$ & $\begin{array}{l}0.40(- \\
0.10- \\
0.907)\end{array}$ & 0.114 & \\
\hline Nursing & 23 & $\begin{array}{l}10.4(9.7- \\
11.2)\end{array}$ & $\begin{array}{l}\text { Social work } \\
(0.001)\end{array}$ & $\begin{array}{l}11.2(10.3- \\
12.1)\end{array}$ & $\begin{array}{l}0.78(- \\
0.24-1.8)\end{array}$ & 0.125 & \\
\hline Pharmacy & 71 & $\begin{array}{l}9.6(9.2- \\
10.0)\end{array}$ & $\begin{array}{l}\text { Social work } \\
(0.015)\end{array}$ & $\begin{array}{l}9.7(9.3- \\
10.2)\end{array}$ & $\begin{array}{l}0.16(- \\
0.34-0.65)\end{array}$ & 0.533 & \\
\hline $\begin{array}{l}\text { Social } \\
\text { Work }\end{array}$ & 19 & $\begin{array}{l}8.1(7.3- \\
8.8)\end{array}$ & & $\begin{array}{l}9.3(8.5- \\
10.2)\end{array}$ & $\begin{array}{l}1.26(0.24- \\
2.30)\end{array}$ & 0.019 & \\
\hline Therapist & 5 & $\begin{array}{l}12.2(9.9- \\
14.5)\end{array}$ & $\begin{array}{l}\text { Pharmacy } \\
(0.001)\end{array}$ & $\begin{array}{l}13.0(11.6- \\
14.4)\end{array}$ & $\begin{array}{l}0.80(- \\
3.35-4.96)\end{array}$ & 0.621 & \\
\hline $\begin{array}{l}\text { Previous } \\
\text { IPE } \\
\text { projects }\end{array}$ & & & 0.444 & & & & 0.225 \\
\hline Yes & 112 & $\begin{array}{l}9.9(9.5- \\
10.3)\end{array}$ & & $\begin{array}{l}10.2(9.8- \\
10.6)\end{array}$ & $\begin{array}{l}0.35(- \\
0.71-0.77)\end{array}$ & 0.103 & \\
\hline
\end{tabular}




\begin{tabular}{|c|c|c|c|c|c|c|c|}
\hline Group & No. & $\begin{array}{l}\text { Pre- } \\
\text { intervention } \\
\text { group score } \\
\text { (mean, } \\
95 \% \mathrm{Cl} \text { ) }\end{array}$ & $\begin{array}{l}\text { P-value } \\
\text { (comparison } \\
\text { between } \\
\text { groups for } \\
\text { pre- } \\
\text { intervention } \\
\text { score) }\end{array}$ & $\begin{array}{l}\text { Post- } \\
\text { intervention } \\
\text { group score } \\
\text { (mean, } \\
95 \% \mathrm{Cl} \text { ) }\end{array}$ & $\begin{array}{l}\text { Mean } \\
\text { difference } \\
\text { between } \\
\text { post minus } \\
\text { pre } \\
\text { intervention } \\
\text { scores } \\
\text { (mean, } \\
95 \% \text { cl) }\end{array}$ & $\begin{array}{l}\text { P-value for } \\
\text { mean } \\
\text { difference } \\
\text { between } \\
\text { post minus } \\
\text { pre } \\
\text { intervention } \\
\text { scores }\end{array}$ & $\begin{array}{l}\text { P-value for } \\
\text { comparison } \\
\text { between } \\
\text { pre and } \\
\text { post } \\
\text { intervention } \\
\text { group } \\
\text { scores }\end{array}$ \\
\hline No & 63 & $\begin{array}{l}9.5(9.0- \\
9.9)\end{array}$ & & $\begin{array}{l}10.1(9.7- \\
10.6)\end{array}$ & $\begin{array}{l}0.65(0.18- \\
1.12)\end{array}$ & 0.007 & \\
\hline $\begin{array}{l}\text { Activities } \\
\text { Outside } \\
\text { Faculty }\end{array}$ & & & 0.162 & & & & 0.149 \\
\hline Yes & 40 & $\begin{array}{l}9.3(8.8- \\
9.9)\end{array}$ & & $\begin{array}{l}9.7(9.0- \\
10.4)\end{array}$ & $\begin{array}{l}0.38(- \\
0.32-1.07)\end{array}$ & 0.281 & \\
\hline No & 135 & $\begin{array}{l}9.9(9.5- \\
10.2)\end{array}$ & & $\begin{array}{l}10.3(10.0- \\
10.7)\end{array}$ & $\begin{array}{l}0.48(0.12- \\
0.84)\end{array}$ & 0.009 & \\
\hline
\end{tabular}

Table 8

RIPLS (Pearson Correlations)

\begin{tabular}{|lllll|}
\hline & \multicolumn{2}{l}{ Baseline vs. Post-intervention scores } & \multicolumn{2}{l|}{ Baseline vs. Change in scores } \\
\hline & $r$ & $p$ & $r$ & $p$ \\
\hline Total RIPLS & 0.547 & $<0.001$ & -0.459 & $<0.001$ \\
\hline Teamwork and Collaboration & 0.492 & $<0.001$ & -0.486 & $<0.001$ \\
\hline Professional Identity & 0.505 & $<0.001$ & -0.483 & $<0.001$ \\
\hline Roles and Responsibilities & 0.432 & $<0.001$ & -0.504 & $<0.001$ \\
\hline
\end{tabular}

\section{Change in RIPLS Score Post-intervention}

For all undergraduates, there was a significant decrease in post-intervention RIPLS score (mean difference - 1.3, $-2.5--0.089, p=0.035)$. There was a significant decrease in RIPLS score post-intervention for the following subgroups: male, early years, medical and current or past involvement in activities outside faculty. (Table 4)

For the "teamwork" subscale, there was no significant difference between the pre- and post-RIPLS score. (Table 5)

For the subscale "professional identity", there was a significant decrease in the post-intervention score (mean difference $-1.4,-2.1--0.77, \mathrm{p}<0.001)$. This was seen in the following subgroups: both genders, early years, both previous involvement and non-involvement in IPE projects, and current or past participation in activities outside of the faculty. (Table 6)

For the subscale "roles and responsibility", there was a significant increase in the post-intervention score (mean difference $0.46,0.14-0.77, p=0.005$ ). This was seen in the following subgroups: female, later years, social work, no previous involvement in IPE projects and current or past participation in activities outside of the faculty.

(Table 7) 
Pearson correlation performed on baseline versus post-intervention scores showed a significant positive correlation for Total RIPLS $(r=0.55, p<0.001)$, as well as all the subscales of Teamwork and Collaboration $(r=$ $0.49, p<0.001)$; Professional Identity $(r=0.51, p<0.001)$; and Roles and Responsibilities $(r=0.43, p<0.001)$. Conversely, there was a significant negative correlation between baseline versus change in scores for Total RIPLS $(r=-0.46, p<0.001)$, as well as all the subscales of Teamwork and Collaboration $(r=-0.49, p<0.001)$; Professional Identity $(r=-0.48, p<0.001)$; and Roles and Responsibilities $(r=-0.50, p<0.001)$.

We also analysed the individual items of the RIPLS. (Table 9) Negatively coded statements like "the function of nurses and therapists is mainly to provide support for doctors" (Item 17) and "I am not sure what my professional role will be" (Item 18) showed significant increases in scores post-intervention $(0.23, p=0.005$ and $0.17, p=0.016$ respectively). Other significant findings include a decrease in scores for the statements "shared learning with other health and social care professionals will help me to communicate better with patients and other healthcare professionals" (Item 13) $(-0.14, p=0.013)$, and "shared learning will help to clarify the nature of patient problems (Item 15) $(-0.10, \mathrm{p}=0.034)$.

\section{Table 9: RIPLS (Individual items analysis)}


Item Learning with other students / professionals will make me a more effective

1 member of a health and social care team.

Item Patients would ultimately benefit if health and social care students /

2 professionals worked together to solve patient problems.

Item Shared learning with other health and social care students / professionals

3 will increase my ability to understand clinical problems.

Item Learning with health and social care students before qualification would

4 improve relationships after qualification.

Item Communication skills should be learned with other health and social care

5 students.

Item Shared learning will help me to think positively about other healthcare

6 professionals.

Item For small group learning to work, students need to trust and respect each

7 other.

Item Team-working skills are essential for all health and social care students to

8 learn.

Item Shared learning will help me to understand my own limitations.

9

Item I don't want to waste time learning with other health and social care

10* students.

Item It is not necessary for undergraduate health and social care students to learn

11* together.

Item Clinical problem solving skills can only be learnt with students from my own

12* department.

Item Shared learning with other health and social care professionals will help me

13 to communicate better with patients and other healthcare professionals.

Item I would welcome the opportunity to work on small group projects with other

14 health and social care students.

Item Shared learning will help to clarify the nature of patient problems.

15

Item Shared learning before qualification will help me become a better team

16 worker.

Item The function of nurses and therapists is mainly to provide support for

17* doctors.

Item I am not sure what my professional role will be.

$18^{*}$

Item I have to acquire much more knowledge and skills than other health or social 19* care students.

* Questions 10-12 and 17-19 are negatively coded, hence a positive difference in means indicates an improvement in attitude

\section{Self-Reported Feedback on IP Learning}


91.6\% participants agreed that they could "better appreciate the importance of IPC in the care of patients". In addition, $72.8 \%$ saw the importance of the MDMs for their learning. $91.9 \%$ of respondents would recommend the program to their friends.

\section{Qualitative Feedback}

A total of 163 participants responded to the qualitative survey. $34.4 \%$ of respondents were male. $33.1 \%$ of respondents were studying medicine, $12.3 \%$ nursing, $40.5 \%$ pharmacy, $11.0 \%$ Social Work and $3.1 \%$ Therapy. $54.6 \%$ of respondents were in early years of study (Year 1-2). $74.8 \%$ had previous exposure to IPE projects and $11.7 \%$ had current or past participation in activities outside of the faculty.

\section{Theme 1: Learning and teaching one another}

Healthcare undergraduates found value in learning from one another. They shared knowledge and skills gained from their respective curriculum with one another.

I feel more equipped and prepared to teach and learn from other healthcare professionals (21-year-old female third-year medical student)

I have learnt a lot from medical students on diagnosis and clinical presentation (22-year-old female fourth-year pharmacy student)

I learnt a lot from my social work team leader and how to consider the social aspects of issues the elderly face (20-year-old male first-year medical student)

\section{Theme 2: Understanding the role of other healthcare professionals}

Healthcare undergraduates learned the role of other healthcare professionals and gained new insights into how different healthcare professionals contributed to the care of the patient.

... learn ... how we can tap on each other strengths to come up with a care plan for the patients (21-year-old female third-year pharmacy student)

It has allowed me to better understand the roles played by other health care professionals (20-year-old female second-year pharmacy student)

Understanding what medicine, nursing [and] pharmacy does make quite a lot of difference to how we perceive and thus, work with them. (23-year-old female second-year social work student)

\section{Theme 3: Understanding one's own role}

Healthcare undergraduates reported understanding the role they played as a part of a multi-disciplinary team.

I am now more aware of the role and responsibility I have as a healthcare professional. (21-year-old female firstyear pharmacy student)

The program has given me a better understanding of what is required of me as a healthcare professional. (22year-old male second-year pharmacy student) 
Really learnt and appreciate ... working in a multi disciplinary team gave me a feel of how it may be like caring for a patient as a team in my future career. (20-year-old female first-year social work student)

\section{Theme 4: Teamwork}

Healthcare undergraduates appreciated the need for collaboration and teamwork within a multi-disciplinary team. They learned about the importance of compromise.

Working with different people, in terms of personality, faculty, etc - I learnt to give and take and be more understanding towards the others. (21-year-old second-year social work student)

It has allowed me to better understand ... how the different professions can come together to better serve the needs of patients. (20-year-old female second-year pharmacy student)

Working together in a team: a lot of collaboration was needed. (20-year-old second-year occupational therapy student)

\section{Theme 5: Opportunity to meet people from other faculties}

Healthcare undergraduates valued the opportunity to meet people from other faculties.

... allows you to get to know people from other faculties. (21-year-old female second-year medical student)

I got to know seniors in medicine and peers from pharmacy. (20-year-old female first-year nursing student)

It is a very unique experience, having the chance to interact with ... other university students from different healthcare faculties. (20-year-old female second-year pharmacy student)

\section{Theme 6: Factors limiting learning}

Healthcare undergraduates shared various constraints including:

Time constraints

It's very taxing on my time and effort, for example, travelling. (22-year-old female fourth-year pharmacy student)

Unmotivated participants

(Trigen can be) very stressful especially during times when fellow team leaders are busy, and there is a lack of support. (19-year-old female second-year social work student)

Burden of administrative tasks

(There are) too many miscellaneous things to do apart from just conducting the visits. (22-year-old female fourthyear pharmacy student)

Lack of suitable patients

I felt a bit useless as other than companionship, medical help etc. could not be rendered other than vitals monitoring which our patient was already doing herself. So what might help better is to sieve and select patients 


\section{Discussion}

\section{Baseline RIPLS score}

The mean baseline RIPLS score is comparable with a study by Chua et al conducted in Singapore.(26) Chua et al also found higher baseline RIPLS scores for medical undergraduates compared to other groups of

undergraduates, a finding also noted in this study and another done in a culturally similar country. $(26,29)$ This is contrary to other studies which found that medical students had a lower RIPLS score.(30, 31) Chua et al. also found that prior exposure to IPE resulted in significantly higher RIPLS scores.(26) We did not find this in our current study. We hypothesise that with the increasing popularity of IPE programs in the institution $(64.6 \%$ of our participants had previous IPE exposure as compared to $10.6 \%$ of participants in Chua et al.), the heterogenous nature of programs may result in differing efficacy in improving attitudes towards IPE.

In the subscale "teamwork", we found that undergraduates in their later years had a lower baseline score as compared to undergraduates in their early years. We postulate that this could be due to undergraduates with more clinical experiences appreciating the challenges of IPE in actual clinical practice. In the subscale "roles and responsibility", we found that social work undergraduates had the lowest baseline score. This is likely because these undergraduates have minimal exposure to social work in the healthcare setting unless they choose such elective modules in their senior years of study.

For the total RIPLS and subscale scores, there is a consistently significant positive correlation between baseline and post-intervention, yet a consistently significant negative correlation between baseline and change in scores. These findings suggest that undergraduates who had a higher baseline scores may be more idealistic about IPE and have these expectations tempered after experiencing the difficulties surrounding IPE. In addition, there could potentially be a ceiling effect wherein participants who are at baseline more ready for IPE stand to benefit less from the program in this area.

\section{Efficacy of the Program}

There was a significant decrease in the total RIPLS score post-intervention. This was mainly driven by the decrease in the subscale "professional identity" (possible reasons are explored in the next section). There was a significant increase in the subscale "roles and responsibility", spearheaded by significant improvements in the scoring for two negatively coded statements "the function of nurses and therapists is mainly to provide support for doctors" and "I am not sure what my professional role will be". The subscale "teamwork" saw no significant changes.

This was discordant with our quantitative feedback and qualitative feedback which, in contrast, suggested that the undergraduates had improved attitudes towards IPE. In our quantitative self-reported feedback, majority of the undergraduates expressed that they better appreciated the importance of IPC for patient care and many felt that that the MDMs were useful for their learning.

In our qualitative analysis, undergraduates expressed five major themes in their learning pertaining to IPE. Firstly, they shared that they have learned from one another, and are equipped to teach individuals from other professions. This was a major focus on the program as undergraduates were encouraged to share their 
knowledge and skills with team members. Being able to freely learn from and teach one another requires mutual trust and respect which are key elements of collaborative practices.(32) In addition, the undergraduates reported better understanding of their own professional roles and the roles of other healthcare professionals. These two areas are recognised as crucial components of collaborative practice as defined by the Canadian Interprofessional Health Collaborative framework.(32) In this aspect, there is agreement between RIPLS and the qualitative feedback provided as the subscale "roles and responsibility" had a significant increase.

Undergraduates also shared that they learned about teamwork, specifically, conflict resolution and compromise. Finally, undergraduates appreciated the opportunities to meet fellow undergraduates from different faculties. It has been observed in many successful IPE programs that informal social interactions are potentially as important as the actual IPE activities.(33) We observed that the relationships between the undergraduates built over through the program often persisted beyond the end of the program. These relationships can benefit the institution and healthcare system.(6)

\section{Discordance Between RIPLS and Quantitative/qualitative Feedback}

We found the decrease in RIPLS score post-intervention unexpected due to the positive feedback received in the quantitative and qualitative feedback. We postulate a few reasons. Firstly, the RIPLS has been described to have psychometrics issues. Most studies publishing data on exploratory or confirmatory analysis of the RIPLS show much variation in factor structure. As a result, multiple researchers have added or rearranged items and relabel subscales.(34) Secondly, RIPLS is insensitive to course improvements and to pre-post change as suggested by Schmitz et al (2015).(35) We chose the RIPLS at the start of 2014 as it had been widely used and validated and simple to administer. However, during this study, we discovered the potential issues as described above. Thirdly, having gone through a six-month period of fortnightly interaction, undergraduates may have had a better understanding of the challenges of IPE and realities of collaborating within IP teams. Their idealism may have been tampered with a dose of realism. Lestari et al. described how nursing and midwifery undergraduates have lower RIPLS scores as compared to medical and dentistry undergraduates as they had prior clinical experience and likely observed less than ideal interactions amongst members of healthcare teams.(29) Similarly, Makino et al. found that alumni who have studied in an IPE program had a lower mean score on the Modified Attitudes Toward Health Care Teams Scale (ATHCTS) as compared to undergraduates.(36) The authors suggested that the negative attitude of alumni may be due to their professional experience in the context of real-world medicine. Several structural issues in clinical practice have been identified to contribute to this trend, e.g. competition between professionals(37) and power struggles.(38) This could be the reason why a short intervention such as that presented by Chua et al., where undergraduates attend a one-day conference together, could lead to an increase in RIPLS.(26)

Undergraduates rarely have the opportunity to work on real-world problems together and experience the reality and challenges of IPE. Our data seems to support this hypothesis as undergraduates in their later years (with presumptively more real-life experience) had a lower baseline score as compared to undergraduates in their early years. In addition, there was a consistently significant negative correlation between baseline and change in scores in all subscales. Moreover, the qualitative data elicited several practical barriers to IPE, which could have partly accounted for the decrease in RIPLS scores.

\section{Barriers to IPE}

Page 27/32 
Undergraduates reported four main barriers: (1) time constraints, (2) unmotivated team members, (3) burden of administrative tasks, (4) lack of suitable patients. Lack of time as a barrier has been brought up by various authors. $(39,40)$ As this program is voluntary, undergraduates are taking time off their already packed curriculum to participate. We are working with faculties to allow accumulation of academic credits from participation in the program. Participants also cited unmotivated team members as reason for their negative experience. This challenge is mitigated by the voluntary nature of participation. Further measures include more stringent selection of participants and faculty intervention for unmotivated team members. As participants were contributing to clinical care, they were required to document their visits. Multiple studies showed that physicians deemed documentation using electronic health records and administrative work burdensome and excessive time spent on these may be associated with physicians' burnout. $(41,42)$ With feedback from participants regarding the burden of documentation, we are working to streamline and reduce the administrative requirements. Lastly, participants cited the lack of suitable patients to care for and to learn about IPC. This reflected the importance of choice and fit of the patients for the program as well.

\section{Strengths and Limitations}

The strength of our study lies in the use of established evaluation framework (Kirkpatrick). In addition, the sample size is large. Also, this a multimethod study approach where both quantitative and qualitative methods contribute to an integrated inference for complex program evaluation.(22) The limitations of our study include it being single-institution. The participants are volunteers and a self-selected group. Hence, results may not be generalisable. In addition, there was no control arm for the intervention.

\section{Conclusion}

We demonstrated the feasibility and acceptability of an interprofessional, longitudinal, student-initiated home visit program that may improve attitudes towards one's role and responsibilities in interprofessional care for future IPCs.

\section{Abbreviations}

IP - Interprofessional; IPE - Interprofessional education; IPC - Interprofessional collaboration; TriGen - TriGenerational HomeCare; SS - Secondary School; KTPH - Khoo Teck Puat Hospital; NWCDC - North West Community Development Council; RIPLS - Readiness for Interprofessional Learning Scale; GDP - Gross Domestic Product; FA - Frequent Admitters; MOH - Ministry of Health; US - United States; AIP - Aging-in-Place Program; H2H - Hospital-to-Home Program; NUS YLLSoM - National University of Singapore Yong Loo Lin School of Medicine; ANOVA - Analysis of Variance; SPSS - Statistical Package for Social Sciences; Year 1-2 Early years of study (in their respective undergraduate course); Year 3-5 - Later years of study (in their respective undergraduate course); ATHCTS - Attitudes Toward Health Care Teams Scale

\section{Declarations}

Ethical approval and consent to participate:

The institutional review board of National University of Singapore (B-15-272) granted ethical approval for this study. 
Consent for publication:

Written consent was obtained from all healthcare undergraduates who participated in this study.

Availability of data and materials:

The datasets used and/or analysed during the current study are available from the corresponding author on reasonable request.

Competing interests: None.

Funding/Support:

National University of Singapore, Yong Loo Lin School of Medicine, Dean's Office; the North West Community Development Council; Khoo Teck Puat Hospital, Singapore provided funding support for the purchase of medical consumables, refreshments and logistics for the program.

Acknowledgements:

The authors would like to thank the Tri-Generational HomeCare Organizing Committee from 2014 to 2018 for supporting the study. They would like to extend their thanks to the National University of Singapore, Yong Loo Lin School of Medicine, Dean's Office; the North West Community Development Council; Khoo Teck Puat Hospital, Singapore; Geriatric Education and Research Institute, Singapore. Finally, they would like to thank the volunteers for their generosity and the patients for their hospitality.

Authors Contributions:

KYYN, GYCL, KSY, NHWN, DGDY, CHW, GCHK conceived and designed the study. KYYN, GYCL, KSY, NHWN, DGDY collected and analysed the data. KYYN, GYCL, KSY wrote the manuscript. All authors interpreted the data and were involved in the development, review and approval of the manuscript.

Authors' Information:

Dr Kennedy NG is Senior Resident, Department of Medical Oncology, National Cancer Centre Singapore, Singapore

Dr Gloria LEUNG is House Officer, Department of Internal Medicine, Changi General Hospital, Singapore

Dr Ka Shing YOW is House Officer, Department of General Surgery, Changi General Hospital

Dr Nerice Heng Wen NGIAM is Medical Officer, Department of Internal Medicine, Singapore General Hospital

Dr Dillon Guo Dong YEO is Medical Officer, Department of Internal Medicine, Singapore General Hospital

Dr Angeline Jie-Yin TEY is Medical Officer, Department of Intensive Care Medicine, Sengkang General Hospital, Singapore

Dr Melanie Si Rui LIM is Senior Resident, Department of Paediatrics Medicine, KK Women's and Children's Hospital 
Dr Aaron Kai Wen TANG is Resident, Department of Psychiatry, Institute of Mental Health Singapore

Bi Hui CHEW is Registered Nurse, Tan Tock Seng General Hospital

Celine THAM is Medical Social Worker, Department of Medical Social Services, Ng Teng Fong General Hospital.

Jia Qi YEO is Pharmacist, National Healthcare Group Polyclinics

Dr Tang Ching LAU is Associate Professor, Vice-Dean (Education), Department of Medicine, Yong Loo Lin School of Medicine, National University of Singapore, Vice-Chairman, Medical Board, National University Hospital, Singapore

Dr Sweet Fun WONG is Chief Transformation Officer, Deputy Chairman, Medical Board, Clinical Director, Populational Health and Community Transformation, Khoo Teck Puat Hospital

Dr Chek Hooi WONG is Adjunct Assistant Professor, Health Services and Systems Research, Duke-NUS Medical School, Singapore; Senior Consultant, Department of Geriatric Medicine, Khoo Teck Puat Hospital, Singapore; Deputy Executive Director, Geriatric Education and Research Institute, Singapore

Prof Gerald C H KOH is Professor, Saw Swee Hock School of Public Health, NUS, NUHS.

\section{References}

1. Practice WSGoIEaC. Framework for Action on Interprofessional Education \& Collaborative Practice. In: Office HPNNaM, editor.: World Health Organisation; 2010.

2. Malone D, Newron-Howes G, Simmonds S, Marriot S, Tyrer P. Community mental health teams (CMHTs) for people with severe mental illnesses and disordered personality. The Cochrane database of systematic reviews. 2007(3):Cd000270.

3. Morey JC, Simon R, Jay GD, Wears RL, Salisbury M, Dukes KA, et al. Error reduction and performance improvement in the emergency department through formal teamwork training: evaluation results of the MedTeams project. Health services research. 2002;37(6):1553-81.

4. Sommers LS, Marton KI, Barbaccia JC, Randolph J. Physician, nurse, and social worker collaboration in primary care for chronically ill seniors. Arch Intern Med. 2000;160(12):1825-33.

5. Holland R, Battersby J, Harvey I, Lenaghan E, Smith J, Hay L. Systematic review of multidisciplinary interventions in heart failure. Heart. 2005;91(7):899-906.

6. Hoffman SJ, Rosenfield D, Gilbert JH, Oandasan IF. Student leadership in interprofessional education: benefits, challenges and implications for educators, researchers and policymakers. Medical education. 2008;42(7):654-61.

7. Harden RM. Interprofessional education: The magical mystery tour now less of a mystery. Anatomical sciences education. 2015;8(4):291-5.

8. Hugh Barr IK, Scott Reeves M, Hammick. Della Freeth. Effective Interprofessional Education: Argument, Assumption and Evidence. Barr H: Blackwell Publishing Ltd; 2005.

9. Singapore's healthcare. spending boom in sight as more hospitals open doors. Singapore Business Review. 2018. 
10. Saxena N, You AX, Zhu Z, Sun Y, George PP, Teow KL, et al. Singapore's regional health systems-a data-driven perspective on frequent admitters and cross utilization of healthcare services in three systems. Int J Health Plan Manag. 2017;32(1):36-49.

11. Low LL, Tay WY, Ng MJM, Tan SY, Liu N, Lee KH. Frequent hospital admissions in Singapore: clinical risk factors and impact of socioeconomic status. Singapore Med J. 2018;59(1):39-43.

12. Sevak P, Stepanczuk CN, Bradley KWV, Day T, Peterson G, Gilman B, et al. Effects of a community-based care management model for super-utilizers. Am J Manag Care. 2018;24(11):e365-e70.

13. Brenner J. Reforming Camden's Health Care System - One Patient at a Time. Prescriptions for Excellence in Health Care Newsletter Supplement. 2009;1(5).

14. Penkunas MJ, Chan AWM, Wong CH, de Korne DF, Tan SM, Wong SF. The Role of a Multicomponent HomeHealth Intervention in Reducing Caregiver Stress in Singapore: A Qualitative Study. The Journals of Gerontology: Series B. 2016;73(2):314-25.

15. Bedoya P, Neuhausen K, Dow AW, Brooks EM, Mautner D, Etz RS. Student Hotspotting: Teaching the Interprofessional Care of Complex Patients. Academic medicine: journal of the Association of American Medical Colleges. 2018;93(1):56-9.

16. The National Center for Complex Health and Social Needs. 2020 [cited 202015 March 2020]. Available from: https://www.nationalcomplex.care/offerings/student-hotspotting/.

17. Jacoby B. Building Partnerships for Service Learning. 1 ed: Jossey-Bass; Jun 2003.

18. Jacoby B. Service-learning in higher eudcation: concepts and practices. 1 ed. San Francisco: Jossey-Bass; 1996.

19. Knowles MS. Andragogy in Action: Applying Modern Principles of Adult Learning (The Jossey-Bass Management Series) 1st Edition. Series TJ-BM, editor: Jossey-Bass; 1984.

20. Pettigrew TF, Tropp LR. A meta-analytic test of intergroup contact theory. J Pers Soc Psychol. 2006;90:75183.

21. Hewstone M, Brown R. Contact is not enough: An intergroup perspective on the 'contact hypothesis.'. Contact and conflict in intergroup encounters. Social psychology and society. Cambridge: Basil Blackwell; 1986. pp. 1-44.

22. Cook TD. Post-positivist critical multiplism. R L Shotland MMM, editor: Sage; 1985.

23. Parsell G, Bligh J. The development of a questionnaire to assess the readiness of health care students for interprofessional learning (RIPLS). 1999;33(2):95-100.

24. Horsburgh M, Lamdin R, Williamson E. Multiprofessional learning: the attitudes of medical, nursing and pharmacy students to shared learning. Medical education. 2001;35(9):876-83.

25. Ruebling I, Pole D, Breitbach AP, Frager A, Kettenbach G, Westhus N, et al. A comparison of student attitudes and perceptions before and after an introductory interprofessional education experience. J Interprof Care. 2014;28(1):23-7.

26. Chua AZ, Lo DY, Ho WH, Koh YQ, Lim DS, Tam JK, et al. The effectiveness of a shared conference experience in improving undergraduate medical and nursing students' attitudes towards inter-professional education in an Asian country: a before and after study. BMC medical education. 2015;15:233.

27. Shapiro SS, Wilk MB. An Analysis of Variance Test for Normality (Complete Samples). Biometrika. 1965;52(3/4):591-611. 
28. Antony Bryant KC. The SAGE Handbook of Grounded Theory: SAGE Publications Ltd; 2010.

29. Lestari E, Stalmeijer RE, Widyandana D, Scherpbier A. Understanding students' readiness for interprofessional learning in an Asian context: a mixed-methods study. BMC medical education. 2016;16:179-.

30. de Oliveira VF, Bittencourt MF, Navarro Pinto IF, Lucchetti ALG, da Silva Ezequiel O, Lucchetti G. Comparison of the Readiness for Interprofessional Learning and the rate of contact among students from nine different healthcare courses. Nurse education today. 2018;63:64-8.

31. Aziz Z, Teck LC, Yen PY. The Attitudes of Medical, Nursing and Pharmacy Students to Inter-Professional Learning. Procedia - Social Behavioral Sciences. 2011;29:639-45.

32. Collaborative. CIH. A national interprofessional competency framework. 2010.

33. Reeves S. Community-based interprofessional education for medical, nursing and dental students. Health Social Care in the Community. 2000;8(4):269-76.

34. Mahler C, Berger S, Reeves S. The Readiness for Interprofessional Learning Scale (RIPLS): A problematic evaluative scale for the interprofessional field. J Interprof Care. 2015;29(4):289-91.

35. Schmitz C, Brandt C. BF. The Readiness for Interprofessional Learning Scale: To RIPLS or not to RIPLS? That is only part of the question. J Interprof Care. 2015;29(6):525-6.

36. Makino T, Shinozaki H, Hayashi K, Lee B, Matsui H, Kururi N, et al. Attitudes toward interprofessional healthcare teams: a comparison between undergraduate students and alumni. J Interprof Care. 2013;27(3):261-8.

37. Tremblay D, Drouin D, Lang A, Roberge D, Ritchie J, Plante A. Interprofessional collaborative practice within cancer teams: Translating evidence into action. A mixed methods study protocol. Implementation science: IS. 2010;5:53.

38. Paradis E, Whitehead CR. Louder than words: power and conflict in interprofessional education articles, 1954-2013. Medical education. 2015;49(4):399-407.

39. Alexandraki I, Hernandez CA, Torre DM, Chretien KC. Interprofessional Education in the Internal Medicine Clerkship Post-LCME Standard Issuance: Results of a National Survey. J Gen Intern Med. 2017;32(8):871-6.

40. West C, Graham L, Palmer RT, Miller MF, Thayer EK, Stuber ML, et al. Implementation of interprofessional education (IPE) in 16 U.S. medical schools: Common practices, barriers and facilitators. J Interprof Educ Pract. 2016;4:41-9.

41. Wright AA, Katz IT. Beyond Burnout - Redesigning Care to Restore Meaning and Sanity for Physicians. N Engl J Med. 2018;378(4):309-11.

42. Patel RS, Bachu R, Adikey A, Malik M, Shah M. Factors Related to Physician Burnout and Its Consequences: A Review. Behav Sci (Basel). 2018;8(11):98. 